\title{
Guidoplasty: A Novel Trick to Save the Day- A Case Report
}

Mohd Iqbal Dar ${ }^{1 *}$, Aamir Rashid ${ }^{2}$, Imran Hafeez ${ }^{2}$, Ajaz A. Lone ${ }^{2}$, Hilal A. Rather ${ }^{2}$, Nisar A. Tramboo ${ }^{2}$

${ }^{1}$ Senior Resident, Department of Cardiology, SKIMS Soura Srinagar, J\&K, India

${ }^{2}$ Consultant Cardiology, Department of Cardiology, SKIMS Soura, J\&K, India

DOI: $10.36347 /$ sjmcr.2021.v09i01.002

| Received: 22.12.2020 | Accepted: 02.01.2021 | Published: 06.01.2021

*Corresponding author: Mohd Iqbal Dar

\section{Abstract}

Catheter-based interventions have become the preferred choice of treatment for most of the cardiac ailments world over. Guiding catheters act as conduits for delivering different sophisticated hardware to the target lesion. Malfunction of these conduits can adversely affect the outcome of an interventional procedure. A 60 years old male presented with a history of 4 hours of chest pain. ECG was suggestive of an inferior wall myocardial infarction. Left sided angiogram with JL 4 diagnostic catheter revealed normal coronaries. Right coronary artery had a proximal lesion. After predilatation of the lesion, difficulty was encountered in advancing stent due to development of kinks in the guiding catheter. These kinks were treated with serial dilatation with semi-compliant balloons (Guidoplasty). Following this Guidoplasty, the stent and post dilatation balloon could be advanced and the procedure completed successfully. We conclude that careful balloon dilatation of the guiding catheter can be used to facilitate the delivery of hardware through a kinked guiding catheter.

Keywords: Myocardial infarction, guiding catheter, anomalous origin of coronary artery, kinked Guide, case report. Copyright $(\mathcal{C} 2021$ The Author(s): This is an open-access article distributed under the terms of the Creative Commons Attribution 4.0 International License (CC BY-NC 4.0) which permits unrestricted use, distribution, and reproduction in any medium for non-commercial use provided the original author and source are credited.

\section{INTRODUCTION}

Cardiovascular diseases have evolved into epidemic proportions affecting every race and region of the world [1]. Advances in the cardiovascular medicine have contributed significantly to the attempts to contain the effects of this epidemic affecting mankind. Miniaturization of the hardware has revolutionized the field of percutaneous intervention. Advanced devices can be deployed effectively using appropriate conduits. These conduits play an important part in the overall success of the procedure and their malfunction can adversely affect the procedural outcome. Here, we describe a case of conduit (Guiding catheter) malfunction and its successful management.

\section{Case Presentation}

A 60 years old male presented with a history of 4 hours of chest pain. ECG was suggestive of an inferior wall myocardial infarction. Patient was taken for an urgent percutaneous coronary angiography via right femoral route. Left sided angiogram with JL 4 diagnostic catheter revealed normal coronaries on the left side (Figure 01).

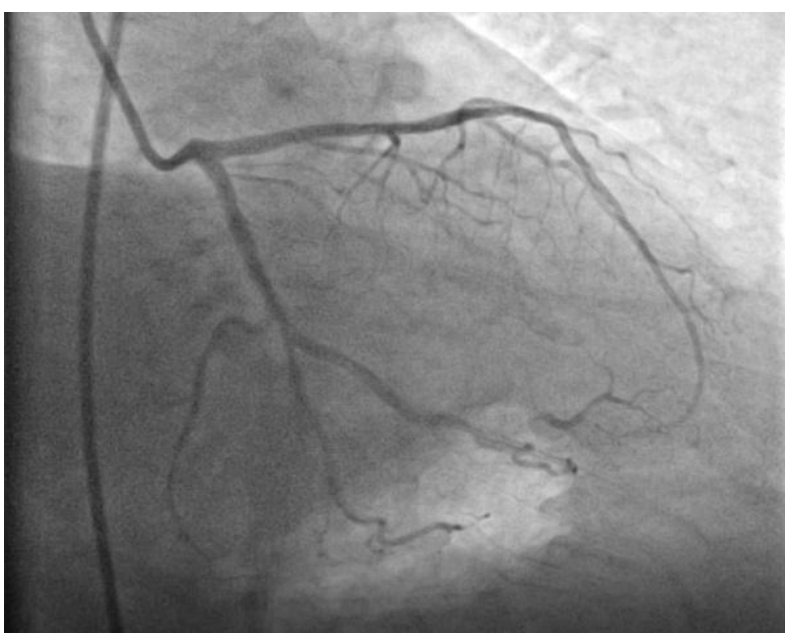

Fig-1: Left sided coronary angiogram.

Multiple attempts to hook the right coronary artery with Judkins right catheter failed. Right coronary artery had anomalous origin with anterior and superior origin. Right coronary artery was hooked with AR1 (Medtronic ${ }^{\circ}$ USA) diagnostic catheter and angiogram showed that the right coronary artery had a significant proximal lesion (Figure 02). 


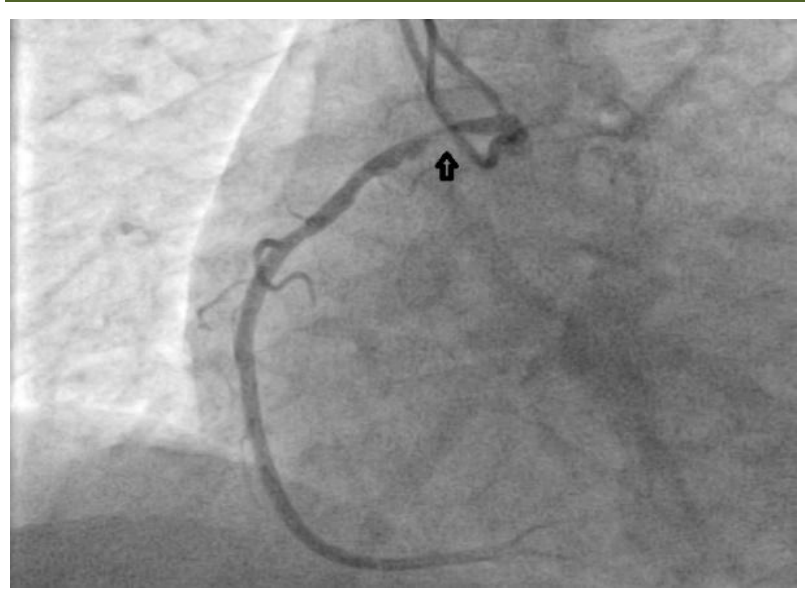

Fig-2: Right coronary angiogram showing proximal lesion (arrow)

Right coronary artery was hooked with difficulty with a JR 4 (Medtronic ${ }^{\circledR}$ USA) guiding catheter after prolonged manipulation of the guide. Lesion was pre-dilated with $2.5 \times 12 \mathrm{~mm}$ SC maverick balloon (Boston Scientific $®$ USA). Some difficulty was noticed while withdrawing the balloon from the guide catheter. A $3.0 \times 28 \mathrm{~mm}$ endeavour resolute stent was chosen for deployment; however, it could not pass forward in the guiding catheter at the level of descending aorta despite multiple attempts. Fall in the aortic pressure tracing was noted on the monitor on manipulation of guiding catheter and advancement of the stent. Careful examination of the guide under fluoroscopy revealed that it had developed two kinks, one at descending aorta level and another at the arch of aorta level. Having already pre-dilated the lesion it was thought to be inconvenient and possibly harmful to withdraw the coronary wire, change the guiding and rewire the pre-dilated coronary artery. After careful consideration of the options, these kinks were treated with serial high-pressure dilatation (Figure 03 and Figure 04) with $2.0 \times 12 \mathrm{~mm} \mathrm{SC}$ and $2.5 \times 12 \mathrm{~mm} \mathrm{SC}$ balloon (Guidoplasty) which facilitated the delivery of the stent and post-dilatation balloon.

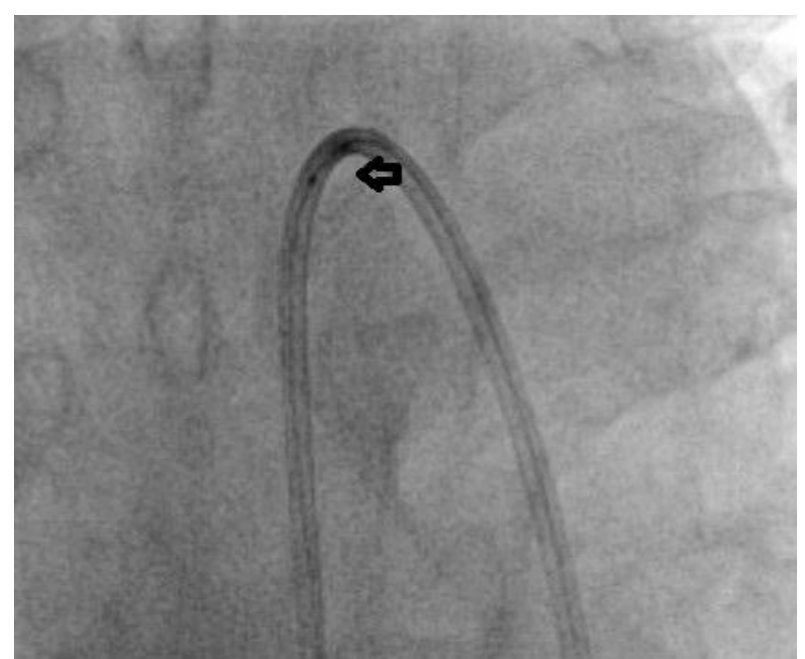

Fig-3: Guidoplasty of the Guiding catheter with a balloon (arrow) at the level of the arch of aorta.

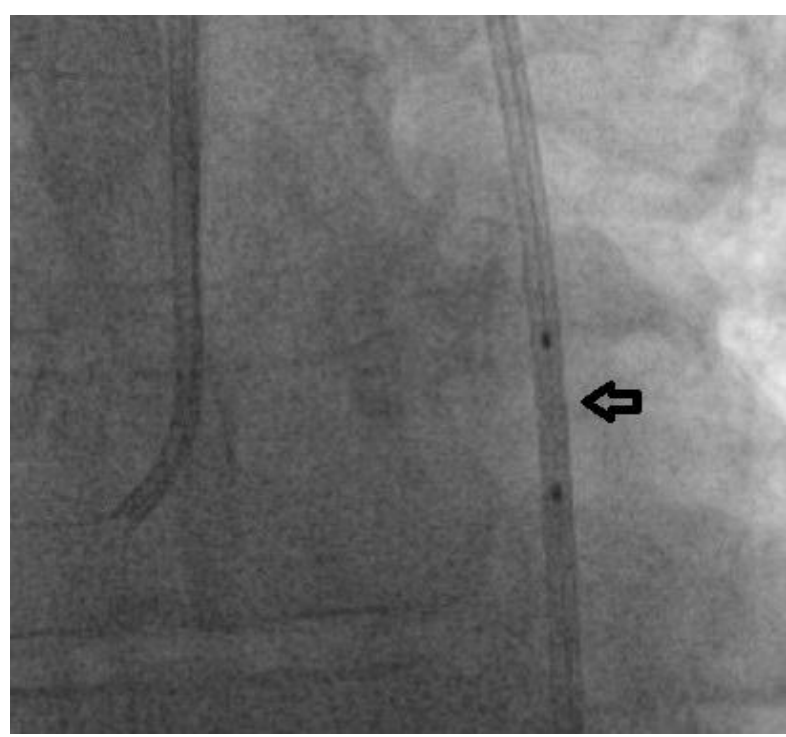

Fig-4: Guidoplasty of guide catheter kink in descending aorta with a balloon (arrow).

(Figure 05).

The procedure was completed successfully

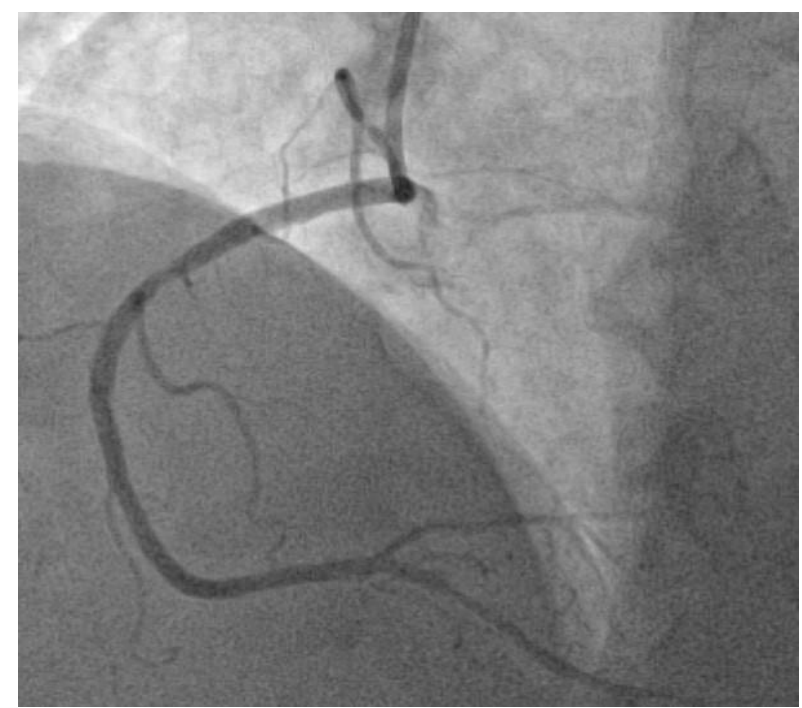

Fig-5: Post-procedure right coronary angiography

\section{DISCUSSION}

Catheter kinks can occur in procedures with femoral and radial access, although more common in procedures with femoral access [2]. Catheter kinks can present as loss or damping of aortic pressure tracing, loss of catheter torque or difficulty in advancing the hardware in the catheter [3]. The major predisposing factor for developing kinks or knots in the catheters are excessive catheter manipulation in attempts to hook a difficult to hook coronary artery or attempts to stabilize or increase the guiding support in an unstable guiding catheter [2, 4]. Management of this conduit failure is conservative in most cases with a gentle opposite turn of the catheter and passing of usual guide wire cause straightening of the catheter [4]. Infrequently invasive management is required to retrieve the catheter in severe entrapment [5]. Conduit malfunction has a 
significant impact on the overall outcome of the procedure.

The current case describes another conservative technique for management of catheter kinks. We used semi-compliant balloons to serially dilate the catheter kinks to facilitate the passage of the stent and post dilatation balloon and successfully complete the procedure. Effective treatment of kinks was marked by continuous aortic tracing on the monitor and easy passage of hardware via the guiding catheter. We recommend this technique when the kinks developed in the middle of the interventional procedure when the change in catheter and other associated hardware is inconvenient, increases the procedure time and may potentially be harmful.

\section{CONCLUSION}

Guidoplasty is a feasible technique to treat guiding catheter kinks and facilitation delivery of hardware for successful completion of an interventional procedure.
Abbreviations

AR 1-Amplatzer Right 1, JR-Judkins Right, JL-Judkins Left

\section{REFERENCES}

1. The global burden of disease: 2013 update.2016. Institute of health metrics and evaluation. USA. http://ghdx.healthdata.org/gbd-data-tool.

2. Pathak L, Shirodkar S, Rajebahadur J, Patil S, Jhavar A, Ruparelia RV. Tips and tricks to manage twisted / kinked diagnostic angiography catheter Tiger catheter (radial route). IHJ Cardiovascular Case Reports (CVCR) 2 (2018). S62-S65.

3. Tanner MA, Ward D. Percutaneous technique for the reduction of knotted coronary catheter. Heart. 2003; 89:1132-1133.

4. Kim JY, Moon KW, Yoo KD. Entrapment of a kinked catheter in the radial artery during transradial coronary angiography. J Invasive Cardiol. 2012; 24:E3eE4.

5. Ul Haq MA, Williams P, Mutha V, Wilson AM, Barlis P. A twist in the transradial coronary catheterisation. Heart Lung Circ. 2014; 23: e84e87. 